\title{
Deep Reinforcement Learning with Adjustments
}

\author{
Hamed Khorasgani, Haiyan Wang, Chetan Gupta, and Susumu Serita \\ Hitachi Industrial AI Lab, Santa Clara, USA
}

\begin{abstract}
Deep reinforcement learning (RL) algorithms can learn complex policies to optimize agent operation over time. RL algorithms have shown promising results in solving complicated problems in recent years. However, their application on real-world physical systems remains limited. Despite the advancements in RL algorithms, the industries often prefer traditional control strategies. Traditional methods are simple, computationally efficient and easy to adjust. In this paper, we first propose a new Q-learning algorithm for continuous action space, which can bridge the control and RL algorithms and bring us the best of both worlds. Our method can learn complex policies to achieve long-term goals and at the same time it can be easily adjusted to address short-term requirements without retraining. Next, we present an approximation of our algorithm which can be applied to address short-term requirements of any pre-trained RL algorithm. The case studies demonstrate that both our proposed method as well as its practical approximation can achieve short-term and long-term goals without complex reward functions.
\end{abstract}

\section{INTRODUCTION}

Optimal control methodologies use system dynamic equations to design actions that minimize desired cost functions. A cost function can be designed to track a trajectory, reach a goal, or avoid obstacles. It is also possible to design a cost function to achieve a combination of goals. Model Predictive Control (MPC) is a common optimal control technique and has been applied to many industrial applications such as pressure control and temperature control in chemical processes [1]. The traditional control solutions are not adequate to address the challenges raised with the evolution of industrial systems. Recently, deep reinforcement learning (RL) has shown promising results in solving complex problems. For example, it has generated superhuman performance in chess and shogi [2]. The following advantages make deep RL a strong candidate to overcome traditional control limitations. First, deep RL has an advantage in solving complex problems, especially when the consequences of an action are not immediately obvious. Moreover, it can learn an optimal solution without requiring detailed knowledge of the systems or their engineering designs. Finally, deep RL is not limited to time-series sensors and can use new sensors such as vision for a better control.

However, deep RL has not been applied to address industrial problems in a meaningful way. There are several key issues that limit the application of deep RL to real-world problems [3]. Deep RL algorithms typically require many samples during training (sample complexity). Sample complexity leads to high computational costs. A high computational cost can be justified for industries as a one-time charge. However, oftentimes small changes in the system goal, such as changing the desired temperature in a chemical reactor, or a new constraint such as a maximum allowable temperature in the reactor, require retraining the model. Moreover, industrial systems often have several short-term and long-term objectives. Designing a reward function that can capture these short-term and long-term goals concurrently can be challenging or even infeasible.

A class of short-term objectives related to safe exploration during RL training have been studied recently. Gu et al. [4] presented an application of deep RL for robotic manipulation control. To ensure safe exploration, they set a sphere boundary for the end-effector position and when the boundaries were about to be violated, they used correction velocity to force the end-effector position back to the center of the sphere. Dalal et al. [5] formulated the safe exploration as an optimization problem. They proposed to add a safety layer that modifies the action at each time step. Toward this end, they learn the constraint function using a linear model and use this model to find the minimal change to the action such that the safety constraints are met at each time step. To the best of our knowledge, there is no study addressing short-term objectives during application.

In this paper, we present a Locally Linear Q-Learning (LLQL) algorithm for continuous action space. The LLQL includes a short-term prediction model, a long-term prediction model, and a controller. The short-term prediction model represents a locally linear model of the dynamic system, while the long-term prediction model represents the value function + a locally linear advantage function. The controller uses the shortterm prediction model and the long-term prediction model to generate actions that achieve short-term and long-term goals simultaneously. It adopts a policy that maximizes Q-value while achieving short-term goals. To make the adoption of our algorithm easier, we propose an approximation version of LLQL which can be applied to any pre-trained RL algorithm. Our solution has the following advantages:

- It does not require designing sensitive reward functions for achieving short-term and long-term goals concurrently.

- It shows better performance in achieving short-term and long-term goals compared to the traditional reward modification methods.

- It is possible to modify the short-term goals without timeconsuming retraining.

- It can be applied to any pre-trained RL model.

The rest of this paper is organized as follows. Section III 
represents the background in dynamic systems and RL. Section IV represents the LLQL algorithm. Section V presents our methodology to achieve short-term and long-term goals using LLQL. Section VI represents LLQL algorithm's approximation for pre-trained RL algorithms. Section VII presents our experimental results. Section VIII presents the conclusions of the paper. Section II discusses the related work.

\section{RELATED WORK}

Our work can be categorized as a new model-based RL approach. Model-based RL algorithms use the environment model which represents the state transition function to plan ahead and select actions that lead to higher rewards. Several model-based algorithms assume the environment model is known. Among them, AlphaZero [2] is one of the most famous. AlphaZero uses the game's rules (Chess, Shogi and Go) as the environment model to generate a series of self-play simulated games. During the simulations, the actions for both players are selected using a Monte-Carlo Tree Search (MCTS) algorithm. The MCTS performs as a planning algorithm by generating candidate actions which are superior to the current policy. The neural network parameters are updated at the end of each game to minimize the game prediction error (loss, draw or win) and maximize the similarity of policy vector to the planning algorithm. AlphaZero is limited to the discrete action space problems. The environment model is typically unknown in real-world applications. Therefore, many model-based RL algorithms learn the state transition model from the data. $N A F$ [9] learns a linear model for state transition at each operating point and uses this model to generate additional samples through imagination rollout. World Models [16] uses a Variational Auto Encoder (VAE) to map a state variable, $x \in X$ to a lower dimensional variable $z$ in a latent space $Z$. It then uses a recurrent neural network (RNN) to learn the state transition model in the latent space. Finally, it applies a simple linear controller to $z$ and the hidden state in the RNN, $h$, to control the system.

Imagination-Augmented Agents (I2As) [10] introduces two paths: 1) model-free path and 2) imagination path. The imagination path learns a transition model and uses this model to generate imagination rollouts. These rollouts are aggregated with the samples in the model-free path. To generate actions in the imagination path, I2As uses the model-free path policy. Therefore, the rollouts in the imagination path improve as the I2As policy improves. Using the imagination rollouts, I2As converge faster than a model-free network with the same number of parameters. [14] showed that a two-step control policy based on 1) learning the dynamic model and 2) applying MPC to the learned model is significantly more sample efficient than model-free RL. However, this approach cannot achieve high rewards. To achieve higher rewards and preserve sample efficiency, they proposed a hybrid model-based and model-free $(M B M F)$ algorithm which runs the model-based approach to achieve the initial result in a sample efficient way, it then trains a model-free policy to mimic the learned model- based controller, and uses the resulting imitation policy as the initialization for the final model-free RL algorithm.

[17] proposed Model-based Value Expansion (MVE) algorithm, which limits the uncertainty in the model by only allowing imagination up to a fixed number of steps, $\mathrm{H}$. MVE uses the learned system dynamic model to generate simulation data up to $\mathrm{H}$ steps into the future, and applies these sample points to estimate Q-function. Instead of saving simulated samples in an imagination buffer, MVE retrains the dynamic model and generates a new imagination rollout at each step. [18] expanded MVE algorithm by proposing Stochastic Ensemble Value Expansion (STEVE), to generate a solution more robust to model uncertainty. [5] proposed safe exploration by modeling constraints using a linear model and applied Lagrangian optimization to modify the action in order to guarantee safety. In this work, we also used Lagrangian optimization for short-term constraints. However, our approach is different in two ways: 1) our method does not modify the RL action to achieve the goals. Instead, it derives an action by considering both long-term goals and short-term constraints. This is possible because our algorithm uses a locally linear model to represent the advantage function. 2) Unlike safe exploration, the focus of this paper is in handling new constraints in the application phase without retraining the model.

\section{BACKGROUND AND DEFINITIONS}

In this section, we review the backgrounds in dynamic systems and reinforcement learning.

\section{A. Dynamic Systems}

A continuous-time dynamic system can be represented as:

$$
\frac{d x(t)}{d t}=f(x(t), u(t), t ; p)
$$

where given the system parameters, $p, f$ maps the state variables, $x \in X$, and actions, $u \in U$, to the state derivative, $\frac{d x}{d t}$ at time $t$. In state space control, the goal is to design a control policy, $\pi_{\text {control }}(u(t) \mid x(t))$, that generates proper actions so as the state variables follow the given desired trajectory, $x_{d}(t)$. It is challenging to design a control policy for a nonlinear complex system represented in equation (1).

The control problem becomes much easier to address when this system is linear with respect to the input [6]. We can present these systems as:

$$
\frac{d x(t)}{d t}=f(x(t))+g(x(t)) u(t) .
$$

Since measurements are typically sampled in discrete times, we derive a discrete time version of linear system (2). Using a first-order approximation as:

$$
x_{k+1}=x_{k}+\Delta\left(f\left(x_{k}\right)+g\left(x_{k}\right) u_{k}\right),
$$

where $x_{k}$ represents variable $x$ at sample point $k . \Delta$ is the sampling rate. In this paper, we assume $\Delta$ is constant. 


\section{B. Reinforcement Learning}

The goal of RL is to learn a policy, $\pi_{R L}\left(u_{k} \mid x_{k}\right)$, that generates a set of actions, $u \in U$, that maximize the expected sum of rewards, $R_{k}=\sum_{i=k}^{T} \gamma^{i-k} r\left(x_{i}, u_{i}\right)$, where $\gamma<1$ is the discount factor, $r$ is the reward function and $T$ represents the end time and can be set to $T=\infty$. The goal is to learn $\pi_{R L}$ for environment, $E_{n}$, such that $\max \left(R=\mathbb{E}_{r_{i}>1, x_{i}>1} \sim E_{n}, u_{i}>1 \sim \pi_{R L}\left[R_{1}\right]\right)$. Unlike control algorithms, model-free reinforcement learning algorithms assume the system dynamic is unknown. Q-function, $Q^{\pi}\left(x_{k}, u_{k}\right)$ is defined as the expected return at state $x_{k}$ when we take action $u_{k}$ and adopt policy $\pi$ afterward: $Q^{\pi}\left(x_{k}, u_{k}\right)=$

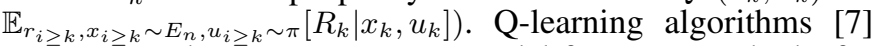
are among the most common model-free RL methods for discrete action space problems. These algorithms use the Bellman recursive equation to model Q-function: $Q^{\mu}\left(x_{k}, u_{k}\right)=$ $\left.\mathbb{E}_{r_{i}>k, x_{i}>k} \sim E_{n}\left[r\left(x_{k}, u_{k}\right)+\gamma Q^{\mu}\left(x_{k+1}, \mu\left(x_{k+1}\right)\right)\right]\right)$, where $\mu$ represents a greedy deterministic policy that selects the action which maximizes Q-value at each step:

$$
\mu\left(x_{k}\right)=\operatorname{argmax}_{u} Q\left(x_{k}, u_{k}\right) .
$$

Q-learning algorithms learn the parameters of the function approximator, $\theta^{Q}$, by minimizing the Bellman error: $\min \left(L\left(\theta^{Q}\right)=\mathbb{E}_{r_{k}, x_{k} \sim E_{n}, u_{k} \sim \beta}\left[\left(Q\left(x_{k}, u_{k} \mid \theta^{Q}\right)-y_{k}\right)^{2}\right]\right), y_{k}=$ $r\left(x_{k}, u_{k}\right)+\gamma Q\left(x_{k+1}, \mu\left(x_{k+1}\right)\right)$, where $y_{k}$ is the fixed target Q-function, and $\beta$ represents the exploration policy.

For continuous action domain problems, it is not trivial to solve equation (4) at each time step. Finding an action to maximize $Q$ which can be a complex nonlinear function is computationally expensive or even infeasible. To address this problem, [8] proposed the Deep Deterministic Policy Gradient (DDPG) algorithm, which learns two networks simultaneously. The critic network learns Q-function by minimizing the Bellman error, and the actor network learns parameters of the policy to maximize the estimated value of $\mathrm{Q}$-function. $\mathrm{Gu}$ et al. [9] proposed Normalized Advantage Function (NAF) Qlearning which formulates the Q-function as the sum of the value function, $V(x)$, and the advantage function, $A(x, u)$.

$$
Q\left(x, u \mid \theta^{Q}\right)=V\left(x \mid \theta^{V}\right)+A\left(x, u \mid \theta^{A}\right),
$$

where

$$
A\left(x, u \mid \theta^{A}\right)=-\frac{1}{2}\left(u-\mu\left(x \mid \theta^{u}\right)\right)^{T} P\left(x \mid \theta^{P}\right)\left(u-\mu\left(x \mid \theta^{u}\right)\right) .
$$

$P\left(x \mid \theta^{P}\right)=L\left(x \mid \theta^{P}\right) L\left(x \mid \theta^{P}\right)^{T}$, where $L\left(x \mid \theta^{P}\right)$ is a lowertriangular matrix. The value function is not a function of action, $u$. Therefore, the action which maximizes advantage function, $A$, maximizes the $Q$ function. $P\left(x \mid \theta^{P}\right)$ is a positivedefinite matrix, and therefore, the action that maximizes the advantage function and the $Q$-function is given by $\mu\left(x \mid \theta^{u}\right)$.

\section{LOCALLY LINEAR Q-LEARNING}

In this section, we propose the LLQL algorithm, which like [8] and [9] can handle continuous action space. Our approach learns short-term and long-term prediction models. Using the long-term and short-term models, a controller generates actions that guide the system toward its short-term and longterm goals. Figure 1 shows our proposed structure to learn the parameters of the short-term and long-term prediction models.

Short-term prediction: consider the nonlinear system presented in equation (3). In this work, we use deep neural networks to estimate system functions, $f\left(x_{k}\right)$, and $g\left(x_{k}\right)$ at

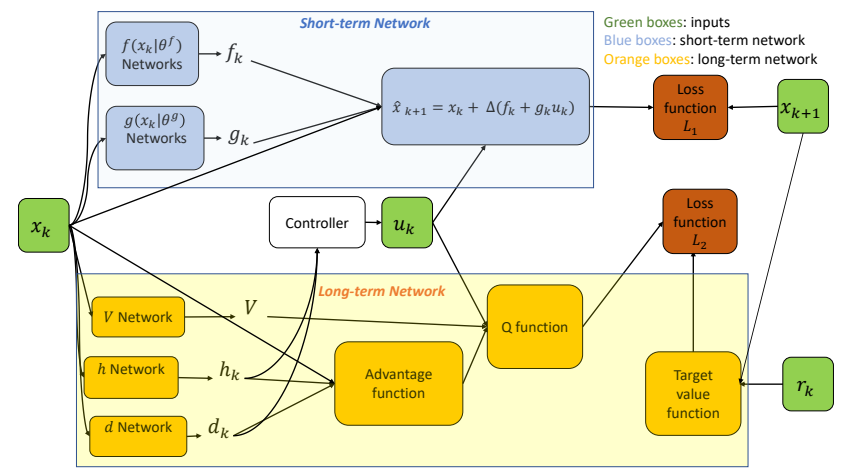

Fig. 1: Learning the LLQL Network Parameters.

each operating point. Substituting the network estimations for these functions in equation (3), we can predict the next state as:

$$
\hat{x}_{k+1}=x_{k}+\Delta\left(f\left(x_{k} \mid \theta^{f}\right)+g\left(x_{k} \mid \theta^{g}\right) u_{k}\right),
$$

where $\hat{x}_{k+1}$ represents our estimation of the next step, and $\theta^{f}$ and $\theta^{g}$ are the network parameters. $\Delta$ is a constant hyper parameter. In dynamic systems, the difference between two consecutive states, $x_{k+1}-x_{k}$, is typically very small. Considering a small $\Delta$ leads to reasonable $f$ and $g$ values and, therefore, improves learning time and accuracy.

We call this dynamic system model short-term prediction model. The controller uses this model to generate actions, which lead the system toward its short-term goals. Note that previous work have used the system short-term dynamic model for generating additional samples in imagination rollout (for example, see [9], and [10]). In this paper, we show that this model can also be used to design actions to achieve short-term goals. To learn the parameters of our short-term prediction model, $\theta^{f}$ and $\theta^{g}$, we minimize the short-term loss function, $L_{1}$, as it is presented in Algorithm 1 .

Long-term prediction: Q-function represents the maximum cumulative reward that can be achieved from current state, $x_{k}$, taking an action $u_{k}$. Therefore, by learning Q-function, we learn the long-term prediction model for the system. Like NAF [9] (see equation (5)), we present Q-function as a sum of value function and advantage function. However, we present the advantage function, $A\left(x, u \mid \theta^{A}\right)$ using a locally linear function of $x_{k}$ and $u_{k}$ as:

$$
\begin{array}{r}
Q\left(x, u \mid \theta^{Q}\right)=V\left(x \mid \theta^{V}\right)+A\left(x, u \mid \theta^{A}\right), \\
A\left(x, u \mid \theta^{A}\right)=-\left\|\left(h\left(x_{k} \mid \theta^{h}\right)+d\left(x_{k} \mid \theta^{d}\right) u_{k}\right)\right\|,
\end{array}
$$

where $h\left(x_{k} \mid \theta^{h}\right)$ and $d\left(x_{k} \mid \theta^{d}\right)$ networks model the locally linear advantage function. Note the NAF advantage function is a special case of the LLQL advantage function when $d\left(x_{k} \mid \theta^{d}\right)=I$, where $I$ represents the identity matrix.

To maximize Q-function, we have to design $u_{k}$ which minimizes $h\left(x_{k} \mid \theta^{h}\right)+d\left(x_{k} \mid \theta^{d}\right) u_{k}$. For simplicity, we present $h\left(x_{k} \mid \theta^{h}\right)$, and $d\left(x_{k} \mid \theta^{d}\right)$ with $h_{k}$ and $d_{k}$ respectively in the remainder of the paper. To maximize Q-function and achieve the long-term goal, we can use simple pseudo-inverse matrix multiplication and derive a solution with the least squares error as:

$$
u_{k}=-\left(d_{k}^{T} d_{k}\right)^{-1} d_{k}^{T} h_{k} .
$$


When $\left\|d_{k}\right\|=0$, it means the network predicts that our action has no impact on the advantage function. Therefore, we choose a random action. Random exploration is an important part of any deep RL algorithm. Therefore, in addition to this unlikely case, we add noise, $\mathcal{N}_{k}$, to the action, $u_{k}$, during the training. We reduce the amount of noise injected to the action as the algorithm converges.

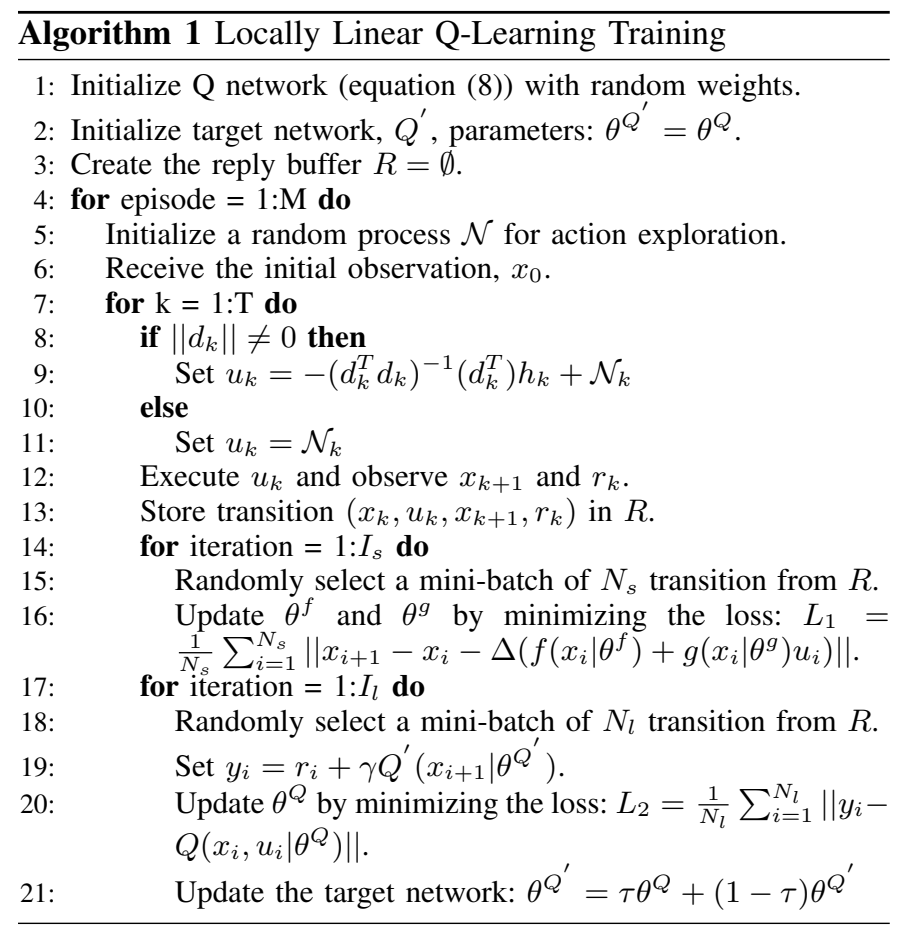

In the application, the controller solves $u_{k}$ with additional constraints to achieve the desired short-term trajectories. We will discuss our short-term adjustment algorithms in the next section. To learn Q-function, in addition to the state estimation error, we minimize the long-term loss function, $L_{2}$, as it is presented in Algorithm 1. Note that having the short-term model, it is straightforward to add imagination rollout to our algorithm to increase sample efficiency. However, improving sample efficiency in RL is not the focus of this work.

\section{Control Strategy}

By separating action design from prediction models, LLQL gives us the freedom to design different control strategies for achieving short-term and long-term goals. Moreover, the linear structure of short-term and long-term models simplifies the control design. Consider the case where LLQL has learned a perfect long-term model for an environment using Algorithm 1 In this case, the optimum solution to achieve the long-term goal is given by equation (9). When we have one or more short-term goals as well, we can formulate the control design as an optimization problem to satisfy both short-term and longterm goals as much as possible.

In this paper, we consider two types of short-term goals: 1) desired trajectory, and 2) constraint. In the first scenario, the agent has a short-term desired trajectory. For example, a car may be required to travel with specific speed during certain periods. In the second scenario, the agent has some limitation for a specific period of time. For example, a car is required to keep its speed below certain thresholds at some periods during the trip. To address the first problem, we add an additional term to the cost function for the short-term goal and solve for the action. We deal with the second problem as a constraint optimization.

\section{A. Short-term trajectory}

Let $x_{d}$ represent our desired short-term trajectory. We develop a control strategy to track $x_{d}$ while pursuing the longterm goals. Using system dynamic functions $f_{k}$ and $g_{k}$, we can define our control optimization problem as:

$$
\text { find } u_{k}\left(\gamma_{1}\left(h_{k}+d_{k} u_{k}\right)^{2}+\gamma_{2}\left(x_{k+1}^{d}-x_{k}-\Delta\left(f_{k}+g_{k} u_{k}\right)\right)^{2}\right) \text {, }
$$

where $x_{k+1}^{d}$ represents the desired trajectory at time $k+1 . \gamma_{1}$ and $\gamma_{2}$ are positive coefficients and can be adjusted to give higher weights to the short-term or long-term goals. Note that in this work, we assume the short-term goals are temporary and when their time expires the system goes to the long-term optimum policy given by 9 . For example, we may require a car to have a specific speed at some specific locations.

We can apply a similar pseudo-inverse matrix multiplication, and derive a solution with the least squares error for 10 , as:

$$
\begin{gathered}
u_{k}^{*}=\left(\left[\begin{array}{c}
\gamma_{1} d_{k} \\
-\gamma_{2} \Delta g_{k}
\end{array}\right]^{T}\left[\begin{array}{c}
\gamma_{1} d_{k} \\
-\gamma_{2} \Delta g_{k}
\end{array}\right]\right)^{-1} \times \\
{\left[\begin{array}{c}
\gamma_{1} d_{k} \\
-\gamma_{2} \Delta g_{k}
\end{array}\right]^{T}\left[\begin{array}{c}
-\gamma_{1} h_{k} \\
\gamma_{2}\left(x_{k}+\Delta f_{k}-x_{k+1}^{d}\right)
\end{array}\right] .}
\end{gathered}
$$

\section{B. Short-term constraint}

The LLQL algorithm provides a framework to design the actions considering different constraints. For safe operation, the agent may have to avoid specific states for a period of time (for example, high speed or locations close to an obstacle). For simplicity, we assume at each moment we only have maximum one constraint on one state variable, $x^{i}$. This is a reasonable assumption, because in physical systems the agent is close to one of the boundaries at any moment in time. When this is not the case, we can define new constraints as a combination of constraints. Consider $c_{k}^{i}$ as the constraint on the state variable, $x^{i}$, at time $k$. We can define the constraint optimization problem for LLQL as:

$$
\begin{aligned}
& \min _{\text {find } u_{k}} \frac{1}{2}\left(h_{k}+d_{k} u_{k}\right)^{2} \\
& \text { such that: } \\
& x_{k+1}^{i} \leq c_{k+1}^{i} .
\end{aligned}
$$

$\frac{1}{2}$ is a coefficient added to simplify the mathematical operation. Using our estimation of the next step, $x_{k+1}^{i}=x_{k}^{i}+\Delta\left(f_{k}^{i}+\right.$ $\left.g_{k}^{i} u_{k}\right)$, we can derive the optimum action which satisfies the constraint as:

$$
u_{k}^{*}=-\left(d_{k}^{T} d_{k}\right)^{-1} d_{k}^{T}\left(h_{k}+\lambda^{*} \alpha_{1}\right),
$$


where $\alpha_{1}=\Delta g_{k}^{i} d_{k}^{T}\left(d_{k} d_{k}^{T}\right)^{-1}, \alpha_{2}=\Delta g_{k}^{i}\left(d_{k}^{T} d_{k}\right)^{-1} d_{k}^{T}$, and $\lambda^{*}=\frac{x_{k}^{i}+\Delta f_{k}^{i}-c_{k+1}-\alpha_{2} h_{k}}{\alpha_{1} \alpha_{2}}$. The derivation details for shortterm constraints are presented in Section A

\section{Vi. Application of LLQL Approximation to the State of the ARt DeEP RL Algorithms}

Deep RL has made significant progress in recent years. Algorithms such as Soft Actor-Critic (SAC) [11], Proximal Policy Optimization (PPO) [12], Asynchronous Advantage Actor Critic (A3C) and Advantage Actor Critic (A2C) [13], have made RL solutions more general, more stable and more sample efficient. In this section, we develop an approximation of LLQL algorithm which can be applied to any pre-trained RL solution. Using this approximation, the users can apply the state-of-the-art RL algorithms + short-term adjustments.

Consider equation (10). The first term in equation (10), $\gamma_{1}\left(h_{k}+d_{k} u_{k}\right)^{2}$, is designed to achieve the long-term goal. When we have a pre-trained RL model, the action generated by the network is designed to achieve the long-term goal. Therefore, we can rewrite equation (10) by replacing the first term in equation $(10)$ by $\gamma_{1}\left(u_{k}-u_{k}^{N}\right)^{2}$, where $u_{k}^{N}$ is the action generated by the pre-trained network. The goal in this optimization problem is to minimize the short-term error and the error between the new action and the action generated by the pre-trained network. Solving this optimization problem, we have:

$$
\begin{gathered}
u_{k}^{*}=\left(\left[\begin{array}{c}
\gamma_{1} \\
-\gamma_{2} \Delta g_{k}
\end{array}\right]^{T}\left[\begin{array}{c}
\gamma_{1} \\
-\gamma_{2} \Delta g_{k}
\end{array}\right]\right)^{-1} \times \\
{\left[\begin{array}{c}
\gamma_{1} \\
-\gamma_{2} \Delta g_{k}
\end{array}\right]^{T}\left[\begin{array}{c}
\gamma_{1} u_{k}^{N} \\
\gamma_{2}\left(x_{k}+\Delta f_{k}-x_{k+1}^{d}\right)
\end{array}\right] .}
\end{gathered}
$$

Note to compute $u_{k}^{*}$ using equation (14), we do not need the long-term network. Similarly, we can replace $\left(h_{k}+d_{k} u_{k}\right)^{2}$ in equation 12 by $\left(u_{k}-u_{k}^{N}\right)^{2}$. The goal of this new optimization problem is to minimize the error between the new action and the action generated by the pre-trained network while satisfying the constraints. The optimum action which satisfies the constraint can be computed as:

$$
u_{k}^{*}=u_{k}^{N}-\lambda^{*} \Delta g_{k}^{i}
$$

where $\lambda^{*}=\frac{x_{k}^{i}+\Delta f_{k}^{i}-c_{k+1}+\Delta g_{k}^{i} u_{k}^{N}}{\Delta g_{k}^{i} \Delta g_{k}^{i T}}$.

\section{EXPERIMENTAL RESULTS}

In this section, we demonstrate the performance of LLQL using Mountain Car with Continuous Action (MountainCarContinuous) from OpenAI Gym ${ }^{1}$. Figure $2 \mathrm{a}$ shows the cumulative rewards during the training for the LLQL, the DDPG, and a model-based reinforcement learning based on MPC presented by [14]. The MPC based solution uses the learned short-term predictive model (system dynamic model) to generate a sequence of actions that maximize the reward over a finite horizon. Figure $2 \mathrm{~b}$ shows that the short-term predictive model estimates future states with high precision. However, optimizing for a finite horizon is a disadvantage for the modelbased solution in achieving long-term goals. Increasing the horizon may improve the long-term performance, but it also

\footnotetext{
${ }_{1}^{1}$ http://gym.openai.com/envs/MountainCarContinuous-v0/
}

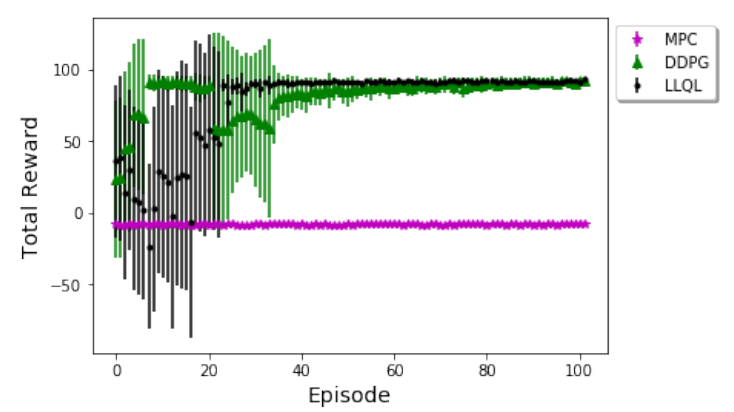

(a) Average and standard deviation of cumulative rewards during the learning process. The experience stops after 1,000 steps or when the car reaches the goal on top of the mountain, car's position $=0.5$, whichever occurs first. For each approach we performed the training 20 times, and selected the top 5 models with the maximum cumulative rewards to calculate mean and standard deviation of each episode.

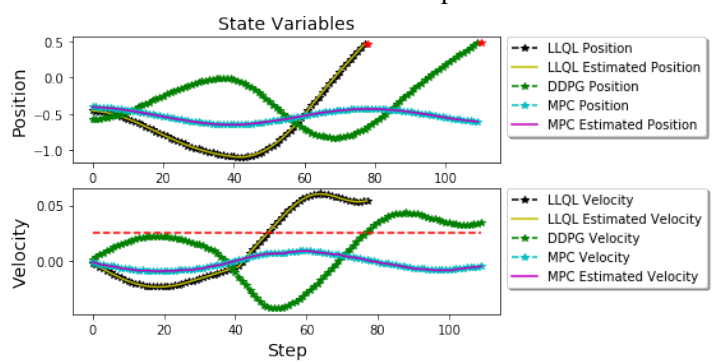

(b) State variables and estimated state variables. Using LLQL model, the car reached the top in 78 steps. Using DDPG model, the car reached the top in 110 steps. The car never reached the top when we used the MPC model. The mean absolute error for position, $\mathrm{x}$, and velocity, $\underline{v}$, estimation in each experiment are: $|\bar{e}|_{x_{L L Q L}}=0.00078,|\bar{e}|_{v_{L L Q L}}=0.000084,|\bar{e}|_{x_{M P C}}=$ $0.00039,|\bar{e}|_{v_{M P C}}=0.00014$. For LLQL algorithm with short-term trajectory we set $\gamma_{1}=1, \gamma_{2}=2000$.

Fig. 2: LLQL for MountainCarContinuous. The network's parameters are presented in Section $\mathrm{B}$

increases the computational costs in the application phase. In our experiments, the car never reached the top of the mountain using the model-based method. Figure $2 \mathrm{~b}$ presents the first 110 steps of a sample experiment. Unlike the MPC based solution, the LLQL and the DDPG algorithms converged in less than 40 episodes (see Figure $2 \mathrm{a}$ ) and reach the top of the mountain in all experiments (see Figure 2b).

\section{A. Short-term trajectory}

Figure $2 \mathrm{~b}$ shows that the policy presented in equation 9 , can lead the car to the top of the mountain using the LLQL algorithm. We can see that the car's velocity is above 0.025 (the red line) when it reaches the top. Now consider the case where we want the car to reach the top of the mountain with our desired velocity, $v_{d}=0.025$. Using equation (11), we can design a control strategy to reach this goal without requiring retraining the LLQL model. We apply the following hybrid control strategy to reach the top of the mountain with our desired speed.

$$
u_{k}= \begin{cases}\text { use equation } 9, & \text { if } x_{k}<0 \\ \text { use equation } 91, & \text { otherwise }\end{cases}
$$




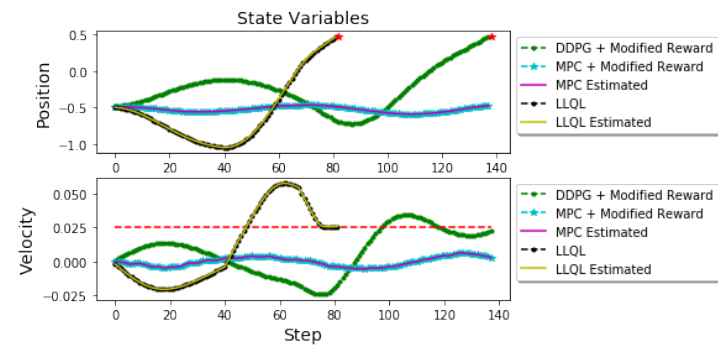

(a) With LLQL the car reached the top of the mountain in 82 steps with a short-term goal error of 0.00008 . With DDPG + modified reward $\left(3^{\text {rd }}\right.$ reward in Table If the car reached the top in 138 steps with short-term goal error 0.0023. The MPC + modified reward cannot guide the car to the top.

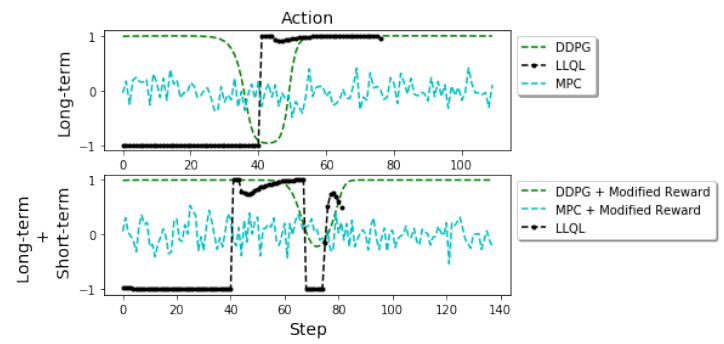

(b) Comparing the actions in normal case versus with the shortterm trajectory. The hybrid strategy (see equation (16) leads to quick adjustment in LLQL action and therefore, smaller shortterm goal error compared to the DDPG + reward modification solution.

Fig. 3: MountainCarContinuous with short-term and long-term goals.

Figure 3a shows that the car can reach the top of the mountain with our desired velocity. When we did not impose our desired speed to the system, the car reached the top of the mountain in 78 steps (see Figure 2b). Demanding a lower speed, slowed down the car and increased the number of steps to 82 (see Figure 3a). Figure $3 \mathrm{~b}$ shows the actions with and without the short-term trajectory. We can see that the action temporarily becomes negative to reduce the velocity to the desired level and then goes back to positive to push the car to the top of the mountain.

TABLE I: Modified Reward functions for short-term trajectory.

\begin{tabular}{||c||}
\hline$r_{t 1}=r_{k}-5000 *\left|v_{k}-v_{d}\right|$ if done \\
\hline$r_{t 2}=r_{k}-100 *\left|v_{k}-v_{d}\right|$ if $x_{k}>0.45$ \\
\hline$r_{t 3}=r_{k}-100 *\left|v_{k}-v_{d}\right|$ if $x_{k}>0.45$ \\
$r_{t 3}=r_{k}-5000 *\left|v_{k}-v_{d}\right|$ if done \\
\hline$r_{t 4}=r_{k}-25000 *\left(v_{k}-v_{d}\right)^{2}$ if done \\
\hline
\end{tabular}

To solve this problem in the traditional way, we had to modify the reward function to achieve both short-term and long-term goals. For comparison, we perform the following experience. We apply DDPG networks and MPC based networks with the modified reward functions shown in Table I Table [ shows that the MPC based solution cannot guide the car to the top (success $=0 / 10$ ). The DDPG with all the modified reward functions can achieve the long-term goal in all the experiences, reaching the top of the mountain in 10 out of 10 experiments.

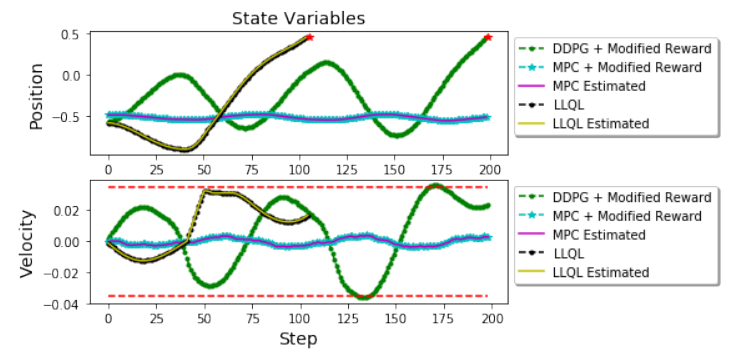

(a) With LLQL the car reached the top in 105 steps without violating the constraints. With DDPG + modified reward $\left(3^{r d}\right.$ reward in Table IV the car reached the top in 199 steps and violated the constraints 13 timesteps. The MPC + modified reward cannot guide the car to the top.

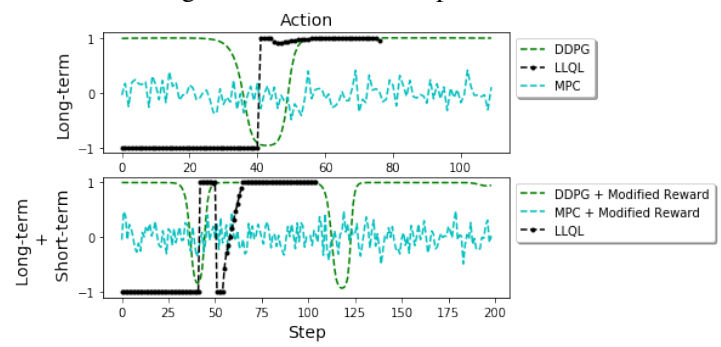

(b) Comparing the actions in the normal case versus with the short-term constraint. The hybrid strategy (see equation (17)) leads to sharp adjustment in LLQL action when the car gets close to the hazardous areas.

Fig. 4: MountainCarContinuous with long-term goal and short-term constraint. The horizontal red lines represent the constraints.

However, the DDPG based solutions do not perform very well with regard to the short-term goal (average absolute velocity error, $|\bar{e}|_{v}$, is too large). On the other hand, LLQL does not require additional training or reward modification, achieves the long-term goal with average steps, $\bar{s}=89.5$, and has the least velocity error, $0.4 \%$.

TABLE II: Short-term trajectory performance

\begin{tabular}{l||l||l||l||l||}
\hline \multicolumn{2}{|l|}{ LLQL Approximation Applied to MountainCarContinuous } & \\
\hline \hline RL Algorithm & modified $r$ & $|e|_{v}$ & $\overline{\mathrm{s}}$ & success \\
\hline$D D P G$ & $r_{t 1}$ & 0.0232 & 109.1 & $10 / 10$ \\
$D D P G$ & $r_{t 2}$ & 0.0193 & 183.6 & $10 / 10$ \\
$D D P G$ & $r_{t 3}$ & 0.0088 & 173.8 & $10 / 10$ \\
$D D P G$ & $r_{t 4}$ & 0.0193 & 103.3 & $10 / 10$ \\
$M P C$ & $r_{t 1}, r_{t 2}, r_{t 3}, r_{t 4}$ & - & 1000 & $0 / 10$ \\
LLQL & - & $\mathbf{0 . 0 0 0 1}$ & $\mathbf{8 9 . 5}$ & $\mathbf{1 0 / 1 0}$ \\
\hline
\end{tabular}

\section{B. Short-term constraint}

Now consider the case where it is unsafe to drive the car above a specific speed, for example, we plan to keep the speed under $v_{k} \leq 0.035$. We can use the following hybrid control strategy to achieve the long-term goal while keeping the speed safe:

$$
u_{k}= \begin{cases}\text { use equation } 9, & \text { if }\left|v_{k}\right| \leq 0.033 \\ \text { use equation } 13, & \text { otherwise. }\end{cases}
$$

We selected the boundary slightly less than the hazardous threshold (0.033 instead of 0.035) to be safe. Figure 4a shows that with the LLQL policy the car reaches its goal while staying outside of hazardous areas. The MPC based solution 


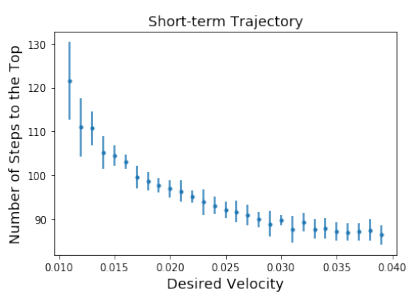

(a) Number of steps to the top vs desired final velocity.

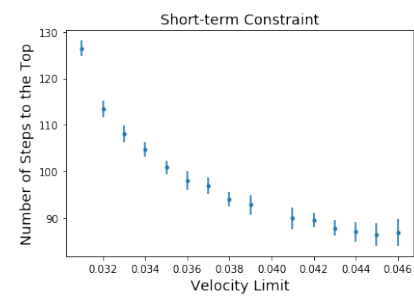

(b) Number of steps to the top vs velocity constraint.
Fig. 5: Long-term performance vs short-term goals. We run the model with each short-term goal 10 times and present the average and standard deviation of the long-term goal.

keeps the car outside of hazardous areas but cannot deliver the long-term goal (reaching the top of the mountain). The DDPG + modified reward reaches the top but fails to deliver the short-term goal (keeping the car out of hazardous areas).

TABLE III: Modified Reward functions for constraint performance.

\begin{tabular}{||c||c||}
\hline \multicolumn{2}{||c||}{$r_{c 1}=r_{k}-10$ if $\left|v_{k}\right|>0.033$} \\
\hline$r_{c 2}=r_{k}-100\left(\left|v_{k}\right|-0.033\right)$ if $\left|v_{k}\right|>0.033$ \\
\hline$r_{c 3}=r_{k}-\left(100\left(\left|v_{k}\right|-0.033\right)\right)^{2}$ if $\left|v_{k}\right|>0.033$ \\
\hline$r_{c 4}=-10$ if $\left|v_{k}\right|>0.033$ \\
\hline
\end{tabular}

TABLE IV: Short-term constraint performance

\begin{tabular}{|c|c|c|c|c|}
\hline \multicolumn{4}{|c|}{ LLQL Approximation Applied to MountainCarContinuous } & \multirow[b]{2}{*}{ success } \\
\hline RL Algorithm & modified $r$ & $\bar{s}_{\text {out }}$ & $\overline{\mathrm{s}}$ & \\
\hline$D D P G$ & $r_{c 1}$ & 21.5 & 100 & $10 / 10$ \\
\hline$D D P G$ & $r_{c 2}$ & 25.2 & 106.8 & $10 / 10$ \\
\hline$D D P G$ & $r_{c 3}$ & 20.4 & 147.5 & $10 / 10$ \\
\hline$D D P G$ & $r_{c 4}$ & 25.1 & 104.1 & $10 / 10$ \\
\hline$M P C$ & $r_{c 1}, r_{c 2}, r_{c 3}, r_{c 4}$ & 0 & 1000 & $0 / 10$ \\
\hline LLQL & - & $\mathbf{0}$ & 98.9 & 10/10 \\
\hline
\end{tabular}

Like the previous section, we apply DDPG network + modified reward function and MPC + modified reward function (see Table III) to compare LLQL with the traditional modelfree and model-based reward engineering approaches. Table IV] shows that unlike LLQL, the modified rewards fail to keep the car below the allowed speed while reaching the top of the mountain. Using equations (11) or (13) for deriving a set of actions is equivalent to solving a sub-optimum solution for the long-term goal in order to satisfy the short-term desired trajectories or constraints. When the short-term goals are far from the global optimum solution, the long-term performance degrades. Figure 5 a shows that lower desired velocities lead to longer traveling time for the MountainCar. Similarly, Figure $5 \mathrm{~b}$ shows that further limiting the maximum velocity degrades the long-term performance.

\section{Application of LLQL Approximation to Pre-trained Deep RL Algorithms}

We use three pertained models from Stable Baselines (SB) [15] for the Pendulum-v0 to show that LLQL's approximation

\footnotetext{
2 http://gym.openai.com/envs/Pendulum-v0/
}

can be used to achieve new adjustments in pre-trained models (see details in Table $\mathrm{V}$, Table $\mathrm{VI}$. For the short-term trajectory control, we set the desired velocity at $v_{d}=0$ when the Inverse Pendulum is vertical, $\cos (x)>0.99$. Note that in this case the short-term trajectory is in harmony with the long-term goal and, therefore, as we can see in Table V even though the short term desired trajectory is improved significantly, the average absolute error, $|\bar{e}|_{v}$, is significantly lower when we apply the adjustment, the average cumulative reward, $\bar{R}$ is not affected in a negative way. For the short-term constraint control, we set the maximum velocity at $v=6$. This is significantly lower than the Inverse Pendulum maximum velocity, $v_{\max }=8$. Table $\mathrm{VI}$ shows that the approximation of the LLQL can be used to satisfy new constraints: number of steps out of speed limit is zero when we apply the adjustment, $\bar{s}_{\text {out }}=0$. In this experiment, we used the same $f$ and $g$ network architectures and learning meta parameters that we used for the MountainCarContinuous.

TABLE V: Short-term trajectory: $v=0$ if $\cos (x)>.99$

\begin{tabular}{|c|c|c|c|c|}
\hline \multicolumn{4}{|c|}{ LLQL Approximation Applied to Pendulum-v0 } & \\
\hline Algorithm & $|e|_{v}$ & $|\bar{e}|_{v}+$ adjustment & $\bar{R}$ & $\bar{R}+$ adjustment \\
\hline SAC 11. & 0.03 & 0.003 & -162 & -151 \\
\hline PPO [12] & 0.16 & 0.008 & -173 & -172 \\
\hline $\begin{array}{lll}\mathrm{A} 2 \mathrm{C} & 13\end{array}$ & 0.03 & 0.005 & -164 & -173 \\
\hline
\end{tabular}

TABLE VI: Short-term constraint: $v<6$

\begin{tabular}{|c|c|c|c|c|}
\hline \multicolumn{4}{|c|}{ LLQL Approximation Applied to Pendulum-v0 } & \\
\hline Algorithm & $\overline{\bar{s}_{\text {out }}}$ & $\bar{s}_{\text {out }}+$ adjustment & $R$ & $R+$ adjustment \\
\hline SAC 11] & 0.40 & 0 & -162 & -170 \\
\hline PPO [12] & 0.31 & 0 & -173 & -184 \\
\hline $\mathrm{A} 2 \mathrm{C}$ & 0.21 & 0 & -164 & -167 \\
\hline
\end{tabular}

\section{CONCLUSIONS}

In this work, we presented LLQL as a new model-based RL algorithm with the capability of achieving both shortterm and long-term goals without requiring complex reward functions. Moreover, we presented an approximation of LLQL which can be applied to any pre-trained RL algorithm. This can be very significant for industrial applications where the RL algorithms have not been used due to the necessity of different short-term adjustments. In the future work, we will investigate conditions where short-term goals are feasible and develop a more analytical approach to set the meta parameters for the controller to guarantee short-term and long-term goals. Moreover, we will model uncertainties in short-term prediction model and apply robust control theory to design robust control solutions.

\section{REFERENCES}

[1] C. E. Garcia, D. M. Prett, and M. Morari, "Model predictive control: theory and practice-a survey," Automatica, vol. 25, no. 3, pp. 335-348, 1989.

[2] D. Silver, T. Hubert, J. Schrittwieser, I. Antonoglou, M. Lai, A. Guez, M. Lanctot, L. Sifre, D. Kumaran, T. Graepel et al., "Mastering chess and shogi by self-play with a general reinforcement learning algorithm," arXiv preprint arXiv:1712.01815, 2017.

[3] G. Dulac-Arnold, D. Mankowitz, and T. Hester, "Challenges of realworld reinforcement learning," arXiv preprint arXiv:1904.12901, 2019. 
[4] S. Gu, E. Holly, T. Lillicrap, and S. Levine, "Deep reinforcement learning for robotic manipulation with asynchronous off-policy updates," in 2017 IEEE international conference on robotics and automation (ICRA). IEEE, 2017, pp. 3389-3396.

[5] G. Dalal, K. Dvijotham, M. Vecerik, T. Hester, C. Paduraru, and Y. Tassa, "Safe exploration in continuous action spaces," arXiv preprint arXiv:1801.08757, 2018.

[6] W.-H. Chen, D. J. Ballance, and P. J. Gawthrop, "Optimal control of nonlinear systems: a predictive control approach," Automatica, vol. 39, no. 4, pp. 633-641, 2003.

[7] C. J. Watkins and P. Dayan, "Q-learning," Machine learning, vol. 8, no. 3-4, pp. 279-292, 1992.

[8] T. P. Lillicrap, J. J. Hunt, A. Pritzel, N. Heess, T. Erez, Y. Tassa, D. Silver, and D. Wierstra, "Continuous control with deep reinforcement learning," arXiv preprint arXiv:1509.02971, 2015.

[9] S. Gu, T. Lillicrap, I. Sutskever, and S. Levine, "Continuous deep qlearning with model-based acceleration," in International Conference on Machine Learning, 2016, pp. 2829-2838.

[10] S. Racanière, T. Weber, D. Reichert, L. Buesing, A. Guez, D. J. Rezende, A. P. Badia, O. Vinyals, N. Heess, Y. Li et al., "Imaginationaugmented agents for deep reinforcement learning," in Advances in neural information processing systems, 2017, pp. 5690-5701.

[11] T. Haarnoja, A. Zhou, P. Abbeel, and S. Levine, "Soft actor-critic: Offpolicy maximum entropy deep reinforcement learning with a stochastic actor," arXiv preprint arXiv:1801.01290, 2018.

[12] J. Schulman, F. Wolski, P. Dhariwal, A. Radford, and O. Klimov, "Proximal policy optimization algorithms," arXiv preprint arXiv:1707.06347, 2017.

[13] V. Mnih, A. P. Badia, M. Mirza, A. Graves, T. Lillicrap, T. Harley, D. Silver, and K. Kavukcuoglu, "Asynchronous methods for deep reinforcement learning," in International conference on machine learning, 2016, pp. 1928-1937.

[14] A. Nagabandi, G. Kahn, R. S. Fearing, and S. Levine, "Neural network dynamics for model-based deep reinforcement learning with model-free fine-tuning," in 2018 IEEE International Conference on Robotics and Automation (ICRA). IEEE, 2018, pp. 7559-7566.

[15] A. Hill, A. Raffin, M. Ernestus, A. Gleave, A. Kanervisto, R. Traore, P. Dhariwal, C. Hesse, O. Klimov, A. Nichol, M. Plappert, A. Radford, J. Schulman, S. Sidor, and Y. Wu, "Stable baselines," https://github.com/ hill-a/stable-baselines 2018.

[16] D. Ha and J. Schmidhuber, "World models," arXiv preprint arXiv:1803.10122, 2018.

[17] V. Feinberg, A. Wan, I. Stoica, M. I. Jordan, J. E. Gonzalez, and S. Levine, "Model-based value expansion for efficient model-free reinforcement learning," arXiv preprint arXiv:1803.00101, 2018.

[18] J. Buckman, D. Hafner, G. Tucker, E. Brevdo, and H. Lee, "Sampleefficient reinforcement learning with stochastic ensemble value expansion," in Advances in Neural Information Processing Systems, 2018, pp 8224-8234.

[19] H. W. Kuhn and A. W. Tucker, "Nonlinear programming," in Traces and emergence of nonlinear programming. Springer, 2014, pp. 247-258.

\section{APPENDIX A}

\section{DERIVATION DETAILS FOR SHORT-TERM CONSTRAINTS}

Consider equation (12). Substituting our estimation of the next step from equation (7) in (12), we have

$$
\begin{aligned}
& \min _{\text {find } u_{k}} \frac{1}{2}\left(h_{k}+d_{k} u_{k}\right)^{2} \\
& \text { such that: } \\
& x_{k}^{i}+\Delta\left(f_{k}^{i}+g_{k}^{i} u_{k}\right) \leq c_{k+1}^{i}
\end{aligned}
$$

Using Lagrangian method at each time step $k$, we have

$$
\begin{aligned}
& L\left(u_{k}, \lambda\right)=\frac{1}{2}\left(h_{k}+d_{k} u_{k}\right)^{2}+ \\
& \lambda\left(x_{k}^{i}+\Delta\left(f_{k}^{i}+g_{k}^{i} u_{k}\right)-c_{k+1}^{i}\right)
\end{aligned}
$$

Taking the gradient of $L$ with respect to $u_{k}$, we can write the Karush-Kuhn-Tucker (KKT) [19] conditions for optimal solution of equation $(18),\left\{u_{k}^{*}, \lambda^{*}\right\}$ as:

$$
\begin{aligned}
& \left(h_{k}+d_{k} u_{k}^{*}\right) d_{k}+\lambda^{*} \Delta g_{k}^{i}=0 \\
& \lambda^{*}\left(x_{k}^{i}+\Delta\left(f_{k}^{i}+g_{k}^{i} u_{k}^{*}\right)-c_{k+1}\right)=0
\end{aligned}
$$

With this assumption, we can show

$$
u_{k}^{*}=-\left(d_{k}^{T} d_{k}\right)^{-1} d_{k}^{T}\left(h_{k}+\lambda^{*} \Delta g_{k}^{i} d_{k}^{T}\left(d_{k} d_{k}^{T}\right)^{-1}\right)
$$

Note that when there is no constraint: $\lambda^{*}=0$, we have $u_{k}^{*}=-\left(d_{k}^{T} d_{k}\right)^{-1} d_{k}^{T} h_{k}$. This is exactly the input we computed in equation (9). When $\lambda^{*} \neq 0$, we have

$$
x_{k}^{i}+\Delta\left(f_{k}^{i}+g_{k}^{i} u_{k}^{*}\right)-c_{k+1}=0
$$

We define $\alpha_{1}=\Delta g_{k}^{i} d_{k}^{T}\left(d_{k} d_{k}^{T}\right)^{-1}$, and $\alpha_{2}=$ $\Delta g_{k}^{i}\left(d_{k}^{T} d_{k}\right)^{-1} d_{k}^{T} . \alpha_{1}$ and $\alpha_{2}$ are scalar. Substituting $u_{k}^{*}$ from equation 21, in equation 22, we have

$$
x_{k}^{i}+\Delta f_{k}^{i}-\alpha_{2}\left(h_{k}+\alpha_{1} \lambda^{*}\right)-c_{k+1}=0
$$

Therefore,

$$
\begin{gathered}
\lambda^{*}=\frac{x_{k}^{i}+\Delta f_{k}^{i}-c_{k+1}-\alpha_{2} h_{k}}{\alpha_{1} \alpha_{2}} \\
u_{k}^{*}=-\left(d_{k}^{T} d_{k}\right)^{-1} d_{k}^{T}\left(h_{k}+\lambda^{*} \alpha_{1}\right) . \\
\text { APPENDIX B } \\
\text { NETWORKS PARAMETERS }
\end{gathered}
$$

In all the networks we shift and scale the state variables to zero mean and unit standard deviation for a better learning. For exploration, we add an additive normal noise to the action. We reduce the noise after each episode with positive cumulative rewards. All the activation functions are Rectified Linear Units (ReLUs). We used the following network structures and parameters in for each model.

- LLQL: $h, d, V, f$ and $g$ networks each has two hidden layers with 200 neurons in each layer. The number of iterations for short-term and long-term prediction model: $I_{s}=I_{l}=5$. The learning rate for the long-term prediction model is 0.001 . The batch size for this model is 10 . The discount rate $\gamma=0.999$. The target model update rate, $\tau=0.001$. The learning rate for the shortterm prediction model is 0.001 for the first 20000 steps and then reduces to $0.0001 . \Delta=0.001$. The batch size for this model is 100 .

- MPC: we use $f$ and $g$ networks for state prediction.

- DDPG: The Q-network, and the deterministic policy network each has two hidden layers with 200 neurons. The learning rate for the Q-network is 0.00001, and the learning rate for the deterministic policy network is 0.000001 . The discount rate $\gamma=0.99$. The batch size is 8. The target model update rate for both networks is 0.1 . 